\title{
Management of Severe COVID-19 with Immunomodulators. Efficacy and Safety Issues
}

\section{Dalia Ashraf ${ }^{1}$, Manar Salem², Norhan Abd El Mohsen³ , Radwa El Borolossy ${ }^{4}$ and Nagwa A Sabri ${ }^{4 *}$}

${ }^{1}$ Inpatient Clinical Pharmacist, Dar El Foad Hospital, Cairo, Egypt

${ }^{2}$ Inpatient Clinical Pharmacist, El Ganzori Hospital, Cairo, Egypt

${ }^{3}$ Inpatient Clinical Pharmacist, El Enayat Hospital, Zagazig, Egypt

${ }^{4}$ Department of Clinical Pharmacy, Faculty of Pharmacy, Ain Shams University,

Cairo, Egypt

*Corresponding Author: Nagwa A Sabri, Department of Clinical Pharmacy, Faculty

of Pharmacy, Ain Shams University, Cairo, Egypt.
Received: June 22, 2021

Published: July 21, 2021

(C) All rights are reserved by Nagwa A Sabri., et al.

\begin{abstract}
The coronavirus disease 2019 (severe acute respiratory syndrome) named as (COVID-19) is a viral replication syndrome accompanied by a host inflammatory response. The cytokine storm and viral escape of cellular immune responses are crucial factors in the aetiology, clinical manifestations, and outcomes of disease. As the illness develops to its later stages, systemic pro-inflammatory cytokines and biomarkers increase, and this correlates with a lower probability of survival. Immune modulators may be able to suppress cytokines and therefore alleviate the cytokine storm. In this review, we will explore the possible function and safety of these medications in the treatment of severe COVID-19, as well as their influence on survival and clinical symptoms.
\end{abstract}

Keywords: Immunomodulatory; COVID; Clinical Response; Side Effects; Mode of Action

\section{Introduction}

Coronaviruses are positive-stranded RNA viruses that have a crown-like appearance (Coronam is the latin term for crown) due to the presence of spike glycoproteins on its envelope. The subfamily Orthocoronavirinae of the Coronaviridae family is classified into four genera: alphacoronavirus, betacoronavirus, deltacoronavirus, and gammacoronavirus. SARS-CoV-2 is a new betacoronavirus that belongs to the same subgenus as SARS-CoV and MERS-CoV, both of which have previously been linked to SARS-CoV and MERS-CoV epidemics with death rates of up to 10 and 35 percent respectively [1].

The coronavirus disease 2019 named as (COVID-19) is a viral replication syndrome accompanied by a host inflammatory re- sponse that is caused by severe acute respiratory syndrome virus (SARS-CoV-2) [2]. The cytokine storm and viral evasion of cellular immune responses are crucial factors in the aetiology, clinical manifestations, and outcomes of disease [3]. As the illness develops to its later stages, systemic pro-inflammatory cytokines and biomarkers increase, and this correlates with a lower probability of survival [4]. Immune modulators may be able to suppress cytokines and alleviate the cytokine storm [5].

Specific immune modulators include cytokines antagonists such as interleukin (IL-1 and -6) receptor antagonists for example; sarilumab, tocilizumab, and anakinra, Janus kinase inhibitors, including, ruxolitinib, and baricitinib, tumor necrosis factor- $\alpha$ antagonist (anti-TNF- $\alpha$ ) agents as infliximab, and adalimumab, granulocyte 
macrophage colony stimulating factors including lenzilumab, gimsilumab, and namilumab, and convalescent plasma with promising to negative trials and other data [6].

On the other hand, non-specific immune modulators includes human immunoglobulin, interferons, corticosteroids as dexamethasone, angiotensin pathway modulators, statins, macrolide antibiotics like (azithromycin, and clarithromycin), colchicine, hydroxychloroquine, chloroquine, and prostaglandin D2 modulators like ramatroban [7].

Dexamethasone $6 \mathrm{mg}$ taken once per day either via oral or intravenous route for 10 days may reduce mortality in COVID-19 patients by one-third, and one-fifth for those on ventilators and for those using oxygen respectively [8]. To maximize treatment results, research efforts should focus not only on the most appropriate immunomodulatory methods, but also on the best timing of such interventions [9].

\section{Immunomodulatory agents}

When a virus enters the body, two lines of immune defense are triggered, which are innate immunity and adaptive immunity. Innate immunity contains a variety of soluble and cell-based antimicrobial agents that are activated very early after infection [10]. Adaptive immunity, which comprises of pathogen-specific antibodies and T-cells, develops later and helps to infection clearance as well as protection against future infections [11].

Immunomodulatory agents can either stimulate (immunostimulants) or depress (immunosuppressants) the immune system, or modulate various aspects of immunity including both the adaptive and innate immunity. These medications are used to boost the immune system's response to infectious diseases. However, in severe covid-19 morbidity and death are linked with hyperinflammation and interference with cytokine signalling [12]. As a result, employing immunomodulatory methods may considerably decrease hyperinflammation in these individuals [13], although immunomodulators' actions can be specific or non-specific [14].

\section{Corticosteroids}

Patients with severe COVID-19 can have a systemic inflammatory response, which can result in lung damage and multisystem organ failure. It has been proposed that the strong anti-inflammatory actions of corticosteroids might avoid or minimize these negative consequences.

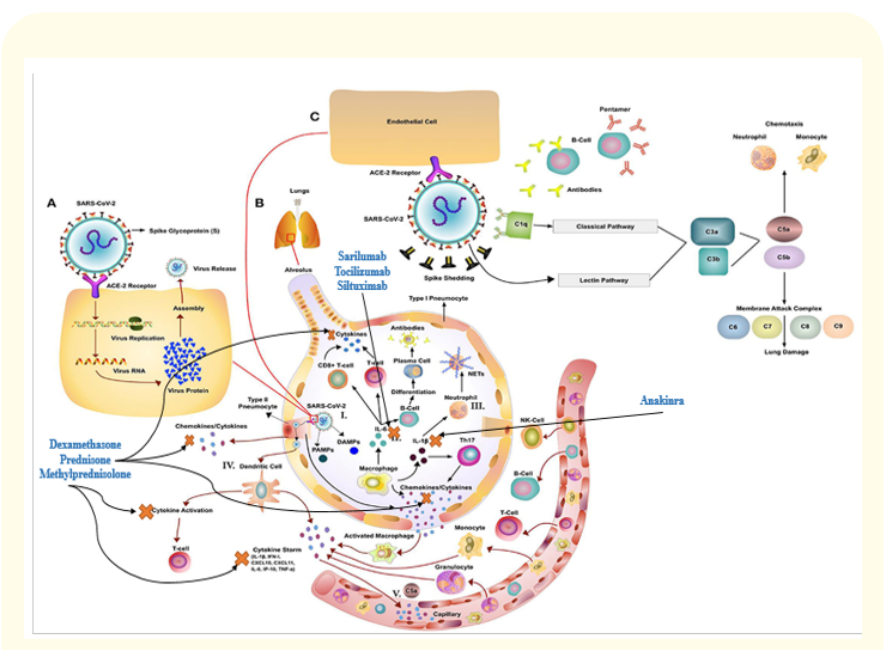

Figure 1: Potential SARS-Cov-2 immune pathogenesis and the effect of different immunomodulators (Prednisone, methylprednisolone, dexamethasone, Anakinra, sarilumab, tocilizumab and siltuximab) on immune response pathways against covid-19 ([15] modified).

\section{Dexamethasone}

A potent and long-acting synthetic glucocorticoid with minimal mineralocorticoid action which approximately possessing 25 times more activity than other glucocorticoids and proved to be beneficial in treatment of COVID-19 patients [16]. Prednisone, methylprednisolone, and hydrocortisone are examples of alternative glucocorticoids that can be used if dexamethasone is not available, moreover, the total daily dose of dexamethasone is equivalent to $6 \mathrm{mg}$ via oral or intravenous route. Glucocorticoids' main antiinflammatory action is to suppress pro-inflammatory genes that encode for cytokines, chemokines, cell adhesions in molecules, and inflammatory response.

It is worthy to mention that, the side effects of dexamethasone include; secondary infection vascular necrosis, hyperglycemia, psychiatric effects, peripheral edema, adrenal insufficiency, myopathy particularly if used with neuromuscular blocking agents long term more than a week could lead to arrhythmia. Prolonged use of systemic corticosteroids may raise the chance of latent infection reactivation and when used during outbreaks of other novel coronavirus infections (MERS and SARS) corticosteroid treatment was associated with delayed virus clearance [17]. 
Corticosteroids (mainly prednisone or methylprednisolone) have been shown to produce both beneficial and negative clinical effects in patients with various lung infections. Prednisone treatment decreased the risk of mortality in individuals with Pneumocystis pneumonia and hypoxia [18]. Besides, it has been linked to delayed virus clearance in outbreaks of other novel coronavirus infections (MERS and SARS) and in severe pneumonia caused by influenza viruses, corticosteroid therapy has been attributed to worse clinical outcomes, including secondary bacterial infection and death [19].

Experimental investigations indicated that inflammatory response decreased following the injection of corticosteroid in severe community-acquired pneumonia [20], consequently, the antiinflammatory action of dexamethasone is assumed to counteract the cytokine storm generated by SARS-CoV-2 infection, therefore protecting the lungs and, ultimately, life. On the other side, it is thought that it may inhibit the innate immune system, reducing viral clearance and worsening evolution [21].

Methylprednisolone appears to alleviate symptoms and lung damage in patients with severe and quickly progressing acute respiratory distress syndrome (ARDS), but treatment does not improve mortality. (1) Lately, early dexamethasone treatment in situations with verified moderate to severe ARDS has been found to improve survival [22].

When compared to standard care alone, intravenous dexamethasone plus standard care resulted in a statistically remarkable increase in the number of days alive and free of mechanical ventilation over 28 days [23].

Recovery trial preliminary findings indicate that dexamethasone decreased mortality by $35 \%$ in ventilated patients and $20 \%$ in patients receiving just oxygen; however, there was no effect for those who did not require respiratory assistance [24].

\section{Interleukin-1 inhibitors ANAKINRA}

Anakinra is a recombinant IL-1 receptor antagonist that is used to treat autoinflammatory diseases. This medication is often delivered subcutaneously to adult patients at a regular dosage of 100 $\mathrm{mg}$ /day [25]. On the other hand, high-dose intravenous anakinra is used off-label to treat hyperinflammatory disorders such as macrophage activation syndrome. Because of the short half-life of anakinra ( $3 \mathrm{~h})$, the medication is generally administered intravenously every 6 hours [26].

Case studies have demonstrated positive responses to anakinra in individuals with high endogenous IL-1, including a survival benefit in patients with sepsis and cytokine storm reversal following tocilizumab failure in adults with macrophage activation syndrome [27], therefore it was considered for patient infected with COVID-19 and high IL-1 levels. Nevertheless, there have been reports of side effects like neutropenia, higher rates of infection with extended combination with tumor necrosis factor-alpha blocking, antibody formation, and injection site irritation. Despite the lack of data, inadvertent first trimester exposure is unlikely to be detrimental [28]. Anakinra, on the other hand, has been widely utilized in the treatment of critically ill infants with rheumatologic disorders, including macrophage activation syndrome.

\section{Interleukin-6 inhibitors}

Interleukin (IL)-6 is a pleiotropic, pro-inflammatory cytokine generated by lymphocytes, monocytes, and fibroblasts. The Food and Drug Administration (FDA) has authorized two types of IL-6 inhibitors: anti-IL-6 receptor monoclonal antibodies as sarilumab, tocilizumab and anti-IL-6 monoclonal antibodies (siltuximab). Relying on facts, infection with the severe acute respiratory syndrome associated coronavirus causes bronchial epithelial cells to produce IL-6 in a dose-dependent manner. Similarly, COVID-19-related systemic inflammation and hypoxic respiratory failure can be linked to increased cytokine release, as seen by higher blood levels of IL-6, C-reactive protein, D-dimer, and ferritin. It is thought that modifying IL-6 levels or its effects may change the course of illness [29].

Both medications resulted in increased liver enzyme levels, which appear to be dosage dependent and increase the risk of severe infections (fungal or bacterial infections). However, bowel perforation occurred only when tocilizumab was administered continuously. Although these molecules are actively carried through the placenta as pregnancy advances, particularly during the third trimester, they may alter immunological responses in the exposed fetus in utero. Furthermore, in children with cytokine release syndrome, only tocilizumab is often utilized following chimeric antigen receptor T-cell treatment [30]. 
Interferon alfa/beta

Interferons are a type of cytokines proved to have both in-vitro and in-vivo antiviral effect, and thus, have been proposed as a possible therapy for COVID-19. Several recombinant interferons (rIFNs) preparations $(-\alpha 2 \mathrm{a},-\beta 1 \mathrm{a},-\alpha 2 \mathrm{~b}$, and $-\beta 1 \mathrm{~b})$ showed in-vitro activity against middle east respiratory syndrome coronavirus [9].

The antiviral effects of IFNs are expressed through activating interferon-stimulated genes [12]. Interferon- $\alpha$ side effects include flu-like symptoms (e.g., myalgia, fatigue, and fever), injection site reactions, decreased blood counts, abnormal liver functions, nausea, vomiting, worsening depression, irritability, insomnia, hypertension, autoimmunity induction [31]. Interferon- $\beta$ side effects presented by flu-like symptoms; myalgia, fatigue, fever, thrombocytopenia, leukopenia, lymphopenia, neutropenia, abnormal liver functions (SGPT > SGOT), injection site reactions, pain, rash, headache, hypertonia, worsening depression, autoimmunity induction [32].

Subtypes of interferon have previously been studied in the treatment of SARS and MERS, where, primary in-vitro studies revealed that IFNs, particularly IFN- $\alpha$ and IFN- $\beta$, have antiviral effects on SARS-CoV [7]. The same results were observed for IFN- $\beta$ in the condition of MERS- $\operatorname{CoV}[8,9]$. Later, in an animal model, IFN- $\beta$ was found to have more antiviral efficacy against MERS-CoV than lopinavir-ritonavir [10].

The effectiveness of IFN- $\beta$ against MERS is currently being studied [11]. Regardless of its antiviral characteristics, IFN- $\alpha$ reduced acute respiratory distress syndrome complications by reducing vascular leakage [13]. Increased expression of a protein known as CD-73 may also contribute to a better prognosis in ARDS. However, these findings were not reproduced in a subsequent trial [14].

Lopinavir and interferons, notably interferon-beta, have been demonstrated to have moderate in-vitro efficacy against SARS-CoV and MERS-CoV, and can be administered in combination with ribavirin [8]. Furthermore, in a common marmoset model, lopinavirritonavir or interferon-beta has been found to decrease viral load and improve lung histopathology [33].

Ribavirin and recombinant interferon therapy has been utilized in the treatment of MERS patients based on past experience with SARS and preclinical evidence [34]. Ribavirin and recombinant in- terferon reduced MERS-CoV multiplication in Vero and LLC-MK2 cells at relatively high doses, but when combined, lower amounts reached equivalent outcomes [35]. High dosages of Ribavirin and recombinant interferon- $\alpha 2 \mathrm{~b}$ given to rhesus macaques 8 hours after MERS-CoV inoculation resulted in lower virus loads and was somewhat effective in preventing pneumonia development compared to untreated animals [36].

According to reviews of the scientific literature, a combination of interferon and ribavirin may be beneficial in individuals with severe MERS-CoV infection [35]. Furthermore, this combination inhibited MERS-CoV in cell culture and appeared to enhance results in an animal investigation [37]. Because both medicines have significant potential side effects, their clinical usage should be carefully weighed against any possible harm [38].

\section{Clinical studies}

Glucocorticoids may have positive benefits in overcoming inflammatory reactions and acute respiratory distress syndrome, making them a conveniently accessible and cost-effective therapeutic option for the COVID-19 epidemic. On the other hand, there are recognized side effects of corticosteroid usage, such as delayed viral clearance, secondary infections, and hyperglycemia [39]. As a result, numerous randomized clinical trials and observational studies on corticosteroids for COVID-19 were begun and shown to be helpful.

A randomized double-blind placebo-controlled trial (metacovid) conducted at one location in Brazil compared the use of shortcourse methylprednisolone $(0.5 \mathrm{mg} / \mathrm{kg}$ twice daily) for 5 days versus placebo in hospitalized patients with confirmed or suspected COVID-19 pneumonia. The adjusted intention-to-treat analysis comprised 393 of 416 individuals [149 participants in methylprednisolone arm and 199 in placebo arm]. At the time of enrolment, $34 \%$ of patients in each group required invasive mechanical ventilation, $51 \%$ in the methylprednisolone group and $45 \%$ in the placebo group required supplementary oxygen. In both groups, the average period from the onset of sickness to randomization was 13 days (Interquartile range 9-16). In terms of 28-day mortality, there was no difference between the groups; 37.1 percent of individuals in the methylprednisolone arm and 38.2 percent in the placebo arm died by day 28 [hazard ratio 0.92 ; 95\% CI, 0.67-1.28; $\mathrm{P}=0.629][40]$. 
Also there was no difference among arms in early mortality at days 7 [16.5 and $23.6 \%$ of participants in the methylprednisolone and placebo arms (Hazard ratio 0.68; 95\% CI, 0.43-1.06; $\mathrm{P}=$ $0.089)]$ and at 14 [27.3 and $31.7 \%$ of participants in the methylprednisolone and placebo arms (Hazard ratio 0.82; 95\% CI, 0.571.18; $\mathrm{P}=0.29)$ ] or in the need for mechanical ventilation by Day 7 [27.3 and $31.7 \%$ of participants in the methylprednisolone and placebo arms (Hazard ratio 0.82; 95\% CI, 0.57-1.18; P = 0.29)]. Participants aged $>60$ years had a reduced death rate on day 28 in the methylprednisolone group than in the placebo group (46.6 versus 61.9\%) [Hazard ratio 0.63: 95\% CI, 0.41-0.98: $\mathrm{P}=0.039$ )]. In terms of safety, there was no statistically significant difference in the requirement for insulin treatment between the methylprednisolone and placebo groups (59.5 versus $49.4 \%$ ) $\mathrm{P}=0.059$ nor in rates of positive blood cultures at day 7 (8.3 versus $8.0 \%$ ) or sepsis until day 28 (38.1 versus $38.7 \%$ ) [40].

An open label multicenter randomized clinical trial conducted in 41 intensive care units in brazil compared the use of intravenous dexamethasone $(20 \mathrm{mg} /$ day for 5 days, then $10 \mathrm{mg} /$ day for 5 days or until discharge from intensive care unit) and standard of care versus the standard of care alone in patients with COVID-19 and moderate to severe acute respiratory distress syndrome. A total of 299 individuals were randomly assigned to either dexamethasone ( $n=151)$ or standard of care treatment $(n=148)$. For both groups, the average duration from symptom start to randomization was nine to ten days, while the average time from mechanical ventilation to randomization was one day. The average duration of dexamethasone treatment was ten days (Interquartile range 6-10 days). At 28 days, the dexamethasone group had a greater mean number of days alive and free of mechanical ventilation than the standard of care group (6.6 versus 4.0 days), estimated discrepancy of 2.3 days. The safety of the dexamethasone and standard of care groups was comparable, the requirement for insulin was 31.1 versus 28.4 percent, and new infections were 21.9 versus 29.1 percent [41].

Furthermore, the observational research comprised 1806 hospitalized COVID-19 patients, 140 of whom were given corticosteroids within 48 hours of their arrival. The early use of corticosteroids was not linked to mortality or the need for mechanical ventilation. Nonetheless, corticosteroid therapy of individuals with an initial C-reactive protein level $\geq 20 \mathrm{mg} / \mathrm{dl}$ was linked with a considerably lower risk of death or mechanical ventilation (Odds ratio, 0.23 ; 95\% CI, 0.08-0.70), while glucocorticoid treatment of patients with C-reactive protein levels $<10 \mathrm{mg} / \mathrm{dl}$ was associated with a significantly increased risk of death or mechanical ventilation (Odds ratio, 2.64; 95\% CI, 1.39-5.03) [42].

In an open-label, multicenter, randomized controlled study (recovery trial), 2104 patients were assigned to receive dexamethasone plus standard treatment, whereas 4321 patients were assigned to standard care. Overall, 454 (21.6\%) of patients given dexamethasone and 1065 (24.6\%) of patients given standard care died within 28 days $(\mathrm{P}<0.001)$. In patients undergoing invasive mechanical ventilation, dexamethasone reduced mortality by onethird (29.0 versus $40.7 \%, \mathrm{p}<0.001$ ), and by one-fifth in patients receiving oxygen without invasive mechanical ventilation (21.5 versus $25.0 \%, p=0.002$ ). In the dexamethasone arm, the probability of progression to invasive mechanical ventilation was lower than in the usual care arm (Relative risk 0.77; 95\% CI, 0.62-0.95) [43].

The World Health Organization conducted a meta-analysis on seven randomized clinical studies. From February 26 to June 9, 2020, 1,703 critically ill patients with COVID-19 were studied in 12 countries. 678 patients got corticosteroids (dexamethasone, hydrocortisone, methylprednisolone), whereas 1,025 received standard treatment or placebo. 95.5 percent of patients on mechanical ventilation $(n=1,559)$ were 60 years old (Interquartile range 52-68 years), including 488 women (28.7 percent). Mortality was assessed after 28 days $(n=5), 21$ days $(n=1)$, and 30 days $(n=$ 1). Corticosteroids group mortality rate was $32.7 \%$ (222 of 678) versus $41.5 \%$ (425 of 1025) in the usual care or placebo group ( $\mathrm{P}$ $<0.001)$.

For patients on mechanical ventilation ( $\mathrm{n}=1559)$; absolute risk for corticosteroids was 30 versus $38 \%$ for usual care or placebo [Odds ratio 0.69 (95\% CI, 0.55-0.86)]. Patients who were not on mechanical ventilation $(n=144)$ had a $23 \%$ absolute risk for corticosteroids versus a $42 \%$ risk for usual treatment or placebo [Odds ratio 0.41 (95\% CI, 0.19-0.88)]. The Recovery study included most of the participants (59\%) in this meta-analysis, and it appears that the advantages seen were primarily related to dexamethasone employed in the Recovery experiment [44].

A multicenter, open-label randomized clinical trial (CORIMUNOANA-1) included patients with coronavirus infection confirmed by reverse transcription polymerase chain reaction. The patients required at least $3 \mathrm{~L} / \mathrm{min}$ of oxygen by mask or nasal cannula but without ventilation assistance. Patients with a score of 5 on the 
WHO Clinical Progression Scale, and a C-reactive protein of more than $25 \mathrm{mg} / \mathrm{L}$ were not required to intensive care unit admission. Eligible patients were randomly allocated (1:1) to either usual treatment plus anakinra or usual care alone. The usual treatment plus anakinra regimen were $200 \mathrm{mg}$ twice a day on days 1 to 3,100 mg twice on day 4, and $100 \mathrm{mg}$ once on day 5 . At day 14, (47\%) of patients in the anakinra group and (51\%) of patients in the usual care group required ventilation or died, with a posterior probability of any anakinra effectiveness (Hazard ratio being less than 1). Serious adverse events occurred in (46\%) of the anakinra group and (38\%) of the usual care group $(p=0 \cdot 45)$ [45].

A retrospective research only included individuals with significant inflammatory characteristics (serum C-reactive protein 100 $\mathrm{mg} / \mathrm{L}$ or ferritin $900 \mathrm{ng} / \mathrm{mL}$, or both). Group 1 received a high dosage $(5 \mathrm{mg} / \mathrm{kg}$ twice a day intravenously), which resulted in a $77 \%$ decrease in mortality at 21 days when compared to a historical control group 2 of COVID-19 patients treated with standard treatment in the same hospital. At 21 days, survival in group 1 was $90 \%$ and in group 2 was $56 \%$ ( $p=0.009)$. Mechanical ventilation-free survival was $72 \%$ in group 1 and $50 \%$ in group $2(p=0.15)$. Bacteremia occurred in $14 \%$ of the group and $13 \%$ of the group. Anakinra discontinuation was not followed by inflammatory relapses [46].

The Ana-COVID research began on March 18, 2020, with a prospective cohort and a retrospective control two arms study, where, patients in the anakinra group were given $100 \mathrm{mg}$ subcutaneously twice daily for 72 hours, then $100 \mathrm{mg}$ daily for 7 days in addition to the conventional therapies as well as supportive care. Admission to intensive care unit for invasive mechanical ventilation or mortality occurred in $(25 \%)$ of the anakinra group versus $(73 \%)$ of the historical group $(\mathrm{p}<0.0001)$. Anakinra's treatment impact remained significant in the multivariate analysis $(p=0.0002)$. An increase in liver aminotransferases was seen in seven (13\%) of the anakinra group and four (9\%) of the historical group [47].

An open-label trial was conducted to evaluate the safety and effectiveness of interleukin (IL-6) blocking with sarilumab in patients with severe COVID-19 pneumonia $\left(\mathrm{PaO}_{2} / \mathrm{FiO}_{2}<300 \mathrm{mmHg}\right)$ and hyper-inflammation with elevated inflammatory markers and serum IL-6 levels Sarilumab $400 \mathrm{mg}$ was given intravenously in addition to conventional care, and the outcomes were compared to those of contemporaneous matched individuals who received standard care alone. The findings on day 28 of follow-up were not statistically different from the comparison group (clinical improve- ment $64 \%$, mortality $18 \% ; \mathrm{p}=$ Non-Significant). Baseline $\mathrm{PaO}_{2} /$ $\mathrm{FiO}_{2}$ ratios were more than $100 \mathrm{~mm} \mathrm{Hg}$, and clinical improvement in individuals with lung consolidation $<17 \%$ was faster following sarilumab (10 days) than after conventional therapy (24 days; $\mathrm{p}=$ 0.01). In both groups, the rates of infection and pulmonary thrombosis were comparable. Sarilumab, on the other hand, was linked with quicker recovery in a subset of individuals who had minimal lung consolidation at baseline [46].

A pilot prospective open single-arm multicenter trial of tocilizumab off-label usage in 63 hospitalized adult patients with severe covid-19. Clinical and laboratory parameters were obtained at baseline day 1, 2, 7, and 14, prospectively. There was no moderate to severe adverse effects associated with tocilizumab. A significant improvement in the levels of ferritin, C-reactive protein, D-dimer occurred. At day 14, the ratio of partial pressure of oxygen $\left(\mathrm{PaO}_{2}\right)$ to fraction of inspired oxygen $\mathrm{FiO}_{2}\left(\mathrm{PaO}_{2} / \mathrm{FiO}_{2}\right)$ improved $(\mathrm{p}<$ 0.05 ). Overall mortality was $11 \%$, and baseline D-dimer levels, but not IL-6 levels, were predictive of death. Taking tocilizumab within 6 days after being admitted to the hospital was linked to a higher chance of survival $(p<0.05)$ [48].

In a retrospective cohort trial that included individuals who required intensive care unit hospitalization and mechanical ventilation tocilizumab was given to individuals who had symptoms of cytokine release syndrome. All of the patients were tracked until they died or were discharged from the hospital. Levels of interleukin- 6 were tested on days $0,3,7,14$, and 21 . Infections were classified as culture positive or culture negative (clinically suspected and treated). The study found that patients who received tocilizumab had greater levels of interleukin- 6 at each measurement (days 0,3 , 7,14 , and 21) than patients who received conventional treatment. On day 7, both groups' interleukin-6 levels peaked. Tocilizumab administration, on the other hand, was linked to an increased risk of infection [49].

In an open-label, randomized study at one site in Iran, individuals with severe COVID-19 were given subcutaneous interferon beta-1a (three times weekly for two weeks). There was no difference within the primary outcome of your time to clinical response between the interferon beta-1a group $(n=42)$ and therefore the control group ( $\mathrm{n}=39$ ). Furthermore, there was no difference between the groups in terms of overall hospital stay, intensive care unit stay, or mechanical ventilation duration. The death rate after 28 days was lower in the interferon beta-1a group; however, four 
patients in the interferon beta-1a group died before receiving the fourth dosage of interferon beta-1a, making interpretation of these data problematic [47].

In a double-blind, placebo-controlled study, non-ventilated patients hospitalized with COVID-19 were administered inhaled interferon beta-1a (once daily for up to 14 days). Those receiving inhaled interferon beta-1a $(n=48)$ were more likely to recover to full ambulation than patients receiving placebo $(n=50)(P=0.04)$, had decreased odds of developing severe disease $(\mathrm{P}=0.046)$, and had less breathlessness. However, more information is needed to fully evaluate these findings [50].

In a retrospective cohort trial conducted in China, 77 people with mild COVID-19 were treated with nebulized interferon alfa$2 \mathrm{~b}$, nebulized interferon alfa-2b plus umifenovir, or umifenovir alone. The time to virus clearance in the upper respiratory tract and decrease in systemic inflammation was quicker in the interferon alfa-2b groups than in the umifenovir alone group. However, the results of this study are difficult to interpret because participants in the interferon alfa-2b with umifenovir group were substantially younger than those in the umifenovir only group (mean age of 40 years in the interferon alfa-2b with umifenovir group versus 65 years in the umifenovir only group) and had fewer comorbidities (15\% in the interferon alfa-2b with umifenovir group versus 54\% in the umifenovir only group) at study entry. The FDA has not authorized the use of nebulized interferon alfa-2b in the United States [49].

\section{Conclusion}

Corticosteroids may be an efficient approach in the care of serious COVID-19 patients with respiratory failure. Dexamethasone has been shown to enhance survival in hospitalized patients who require supplementary oxygen, with the largest advantage shown in individuals who require mechanical ventilation, but there was no benefit in patients with moderate illness.

Up to the presentation of this review, the effectiveness of anakinra or siltuximab in treatment of COVID-19 is limited and more research is urgently needed to reach definitive conclusions.

In hospitalized severely infected COVID-19 adult patients, tocilizumab may be a safe alternative; nevertheless, additional controlled trials in individuals with severe disease are urgently needed to demonstrate the clear benefit with IL-6 target treatment. Rec- ommendations were made against using interferons to treat patients with severe or critical COVID-19, unless in a clinical study, because there is insufficient data to suggest either for or against using interferon beta to treat early ( $<7$ days after symptom start) mild and moderate cases.

\section{Bibliography}

1. Cascella M., et al. "Features, Evaluation, and Treatment of Coronavirus (COVID-19)". In: StatPearls. Treasure Island (FL): StatPearls Publishing (2021).

2. Wu C., et al. "Risk Factors Associated With Acute Respiratory Distress Syndrome and Death in Patients With Coronavirus Disease 2019 Pneumonia in Wuhan, China". JAMA Internal Medicine 180.7 (2020): 934-943.

3. Covid19.who.int. (n.d.). WHO Coronavirus (COVID-19) Dashboard (2021).

4. Cao B., et al. "A Trial of Lopinavir-Ritonavir in Adults Hospitalized with Severe Covid-19". New England Journal of Medicine 382 (2020): 1787-1799.

5. Gao J., et al. "Breakthrough: Chloroquine phosphate has shown apparent efficacy in treatment of COVID-19 associated pneumonia in clinical studies". BioScience Trends 14.1 (2020): 7273.

6. Meyerowitz EA., et al. "Rethinking the role of hydroxychloroquine in the treatment of COVID-19". The FASEB Journal 34.5 (2020): 6027-6037.

7. Cunningham AC., et al. "Treatment of COVID-19: old tricks for new challenges". Critical Care 24.1 (2020).

8. Antonelli G., et al. "Treatment of SARS with human interferons". The Lancet 362.9390 (2003): 1158.

9. Chan JFW., et al. "Broad-spectrum antivirals for the emerging Middle East respiratory syndrome coronavirus". Journal of Infection 67.6 (2013): 606-616.

10. Hart BJ., et al. "Interferon- $\beta$ and mycophenolic acid are potent inhibitors of Middle East respiratory syndrome coronavirus in cell-based assays". Journal of General Virology 95.3 (2014): 571-577.

11. Sheahan TP., et al. "Comparative therapeutic efficacy of remdesivir and combination lopinavir, ritonavir, and interferon beta against MERS-CoV". Nature Communications 11.1 (2020). 
12. Sallard E., et al. "Type 1 interferons as a potential treatment against COVID-19". Antiviral Research 178 (2020): 104791.

13. Bellingan G., et al. "The effect of intravenous interferon-beta1a (FP-1201) on lung CD73 expression and on acute respiratory distress syndrome mortality: an open-label study". The Lancet Respiratory Medicine 2.2 (2014): 98-107.

14. Ranieri VM., et al. "Effect of Intravenous Interferon $\beta-1$ a on Death and Days Free From Mechanical Ventilation Among Patients With Moderate to Severe Acute Respiratory Distress Syndrome: A Randomized Clinical Trial". JAMA 323.8 (2020): 725-733.

15. Ahmed-Hassan Hanaa., et al. "Innate Immune Responses to Highly Pathogenic Coronaviruses and Other Significant Respiratory Viral Infections". Frontiers in Immunology 11 (2020): 1979.

16. Iu D., et al. "A practical guide to the monitoring and management of the complications of systemic corticosteroid therapy". Allergy, Asthma and Clinical Immunology 9.1 (2013): 30.

17. Bozzette SA., et al. "A Controlled Trial of Early Adjunctive Treatment with Corticosteroids for Pneumocystis carinii Pneumonia in the Acquired Immunodeficiency Syndrome". New England Journal of Medicine 323.21 (1990): 1451-1457.

18. Arabi YM., et al. "Corticosteroid Therapy for Critically Ill Patients with Middle East Respiratory Syndrome". American Journal of Respiratory and Critical Care Medicine 197.6 (2018): 757-767.

19. Stockman LJ., et al. "SARS: Systematic Review of Treatment Effects". PLoS Medicine 3.9 (2006): e343.

20. Rodrigo C., et al. "Corticosteroids as adjunctive therapy in the treatment of influenza". Cochrane Database of Systematic Reviews 2.2 (2019).

21. Sibila O., et al. "Effects of glucocorticoids in ventilated piglets with severe pneumonia". European Respiratory Journal 32.4 (2008): 1037-1046.

22. Villar J., et al. "Rationale for Prolonged Corticosteroid Treatment in the Acute Respiratory Distress Syndrome Caused by Coronavirus Disease 2019". Critical Care Explorations 2.4 (2020).

23. Tomazini BM., et al. "Effect of Dexamethasone on Days Alive and Ventilator-Free in Patients With Moderate or Severe Acute Respiratory Distress Syndrome and COVID-19". JAMA 324.13 (2020): 1307-1316.
24. The RECOVERY Collaborative Group. "Dexamethasone in Hospitalized Patients with Covid-19 - Preliminary Report”. New England Journal of Medicine 384 (2020): 693-704.

25. Cavalli G and Dinarello CA. "Treating rheumatological diseases and co-morbidities with interleukin-1 blocking therapies". Rheumatology 54.12 (2015): 269.

26. Grom AA., et al. "Macrophage activation syndrome in the era of biologic therapy". Nature Reviews Rheumatology 12.5 (2016): 259-268.

27. Aouba A., et al. "Targeting the inflammatory cascade with anakinra in moderate to severe COVID-19 pneumonia: case series". Annals of the Rheumatic Diseases 79.10 (2020): 13811382.

28. Flint J., et al. "BSR and BHPR guideline on prescribing drugs in pregnancy and breastfeeding-Part II: analgesics and other drugs used in rheumatology practice: Table 1". Rheumatology 55.9 (2016): 1698-1702.

29. https://www.sanofi.com/en/media-room/press-releases/2020/2020-07-02-22-30-00. (n.d.). Sanofi: Press Releases, Thursday (2020).

30. Gritti G., et al. "IL-6 signalling pathway inactivation with siltuximab in patients with COVID-19 respiratory failure: an observational cohort study". medRxiv (2020).

31. Clark R. “Drug review - Pegasys $\AA$ (peginterferon alfa-2a [40 kDa])". Drugs in Context 4 (2007): 1-20.

32. Chen F., et al. "In vitro susceptibility of 10 clinical isolates of SARS coronavirus to selected antiviral compounds". Journal of Clinical Virology 31.1 (2004): 69-75.

33. Chan JFW., et al. "Treatment With Lopinavir/Ritonavir or Interferon- $\beta 1 \mathrm{~b}$ Improves Outcome of MERS-CoV Infection in a Nonhuman Primate Model of Common Marmoset". Journal of Infectious Diseases 212.12 (2015): 1904-1913.

34. Falzarano D., et al. "Inhibition of novel $\beta$ coronavirus replication by a combination of interferon- $\alpha 2 \mathrm{~b}$ and ribavirin". Scientific Reports 3.1 (2013).

35. Falzarano D., et al. "Treatment with interferon- $\alpha 2 \mathrm{~b}$ and ribavirin improves outcome in MERS-CoV-infected rhesus macaques". Nature Medicine 19.10 (2013): 1313-1317.

36. Peiris JSM., et al. "The Severe Acute Respiratory Syndrome". New England Journal of Medicine 349.25 (2003): 2431-2441. 
37. Al-Tawfiq JA., et al. "Middle East respiratory syndrome coronavirus: a case-control study of hospitalized patients". Clinical Infectious Diseases: An Official Publication of the Infectious Diseases Society of America 59.2 (2014): 160-165.

38. Assiri A., et al. "Epidemiological, demographic, and clinical characteristics of 47 cases of Middle East respiratory syndrome coronavirus disease from Saudi Arabia: a descriptive study". The Lancet Infectious Diseases 13.9 (2013): 752-761.

39. Keller MJ., et al. "Effect of Systemic Glucocorticoids on Mortality or Mechanical Ventilation in Patients With COVID-19". Journal of Hospital Medicine 15.8 (2020): 489-493.

40. Chroboczek T., et al. "Corticosteroids in Patients With COVID-19: What About the Control Group?" Clinical Infectious Diseases 72.6 (2021): 1102-1103.

41. Sterne JAC., et al. "Association Between Administration of Systemic Corticosteroids and Mortality Among Critically Ill Patients With COVID-19". JAMA 324.13 (2020): 1330-1341.

42. "Effect of anakinra versus usual care in adults in hospital with COVID-19 and mild-to-moderate pneumonia (CORIMUNOANA-1): a randomised controlled trial". The Lancet Respiratory Medicine 9.3 (2021): 295-304.

43. Huet T., et al. "Anakinra for severe forms of COVID-19: a cohort study". The Lancet Rheumatology 2.7 (2020): e393-e400.

44. Della-Torre E., et al. "Interleukin-6 blockade with sarilumab in severe COVID-19 pneumonia with systemic hyperinflammation: an open-label cohort study". Annals of the Rheumatic Diseases 79.10 (2020): 1277-1285.

45. Sciascia S., et al. "Pilot prospective open, single-arm multicentre study on off-label use of tocilizumab in patients with severe COVID-19". Clinical and Experimental Rheumatology 38.3 (2020): 529-532.

46. Vazquez Guillamet MC., et al. "Interleukin-6 Trajectory and Secondary Infections in Mechanically Ventilated Patients With Coronavirus Disease 2019 Acute Respiratory Distress Syndrome Treated With Interleukin-6 Receptor Blocker". Critical Care Explorations 3.2 (2021).

47. Davoudi-Monfared E., et al. "A Randomized Clinical Trial of the Efficacy and Safety of Interferon $\beta$-1a in Treatment of Severe COVID-19". Antimicrobial Agents and Chemotherapy 64.9 (2020).

48. Plc S. "Synairgen announces positive results from trial of SNG001 in hospitalised COVID-19 patients". Globe Newswire News Room (2020).
49. Zhou Q., et al. "Interferon- $\alpha 2 b$ Treatment for COVID-19". Frontiers in Immunology 11 (2020): 1061.

50. Hung IFN., et al. "Triple combination of interferon beta-1b, lopinavir-ritonavir, and ribavirin in the treatment of patients admitted to hospital with COVID-19: an open-label, randomised, phase 2 trial". The Lancet (2020).

\section{Volume 5 Issue 8 August 2021}

(C) All rights are reserved by Nagwa A Sabri., et al. 